\title{
¿Cómo entender lo rural en la educación rural?. Una aproximación documental
}

\author{
German Darío Dueñas-García \\ Oscar Espinel ${ }^{2}$
}

\footnotetext{
1. Institución Educativa Haydee Camacho Saavedra german.duenas@uptc.edu.co 2. Corporación Universitaria Minuto de Dios UNIMINUTO
}

Como citar: Dueñas-García, G, \& Espinel, O. (2021). ¿Cómo entender lo rural en la educación rural?. Una aproximación documental. Educación Y Ciencia, (25), e12860. https:// doi.org/10.19053/0120-7105. eyc.2021.25.e12860

\section{Resumen}

El artículo indaga por las concepciones en torno a lo rural y la ruralidad en la educación rural. Para ello, se centra en las publicaciones de los años 2010 a 2020 de la revista Praxis y Saber. A la par, se emplean algunos referentes teóricos con el fin de ampliar los instrumentos de comprensión e indagación. De este modo, lo rural se explora en un contexto sociológico que compara preocupaciones de desarrollo y educación; mientras la ruralidad es abordada como un ambiente más amplio y diferenciado de acuerdo a las experiencias en diferentes territorios.

El rastreo arroja regularidad enunciativa alrededor de cuestiones como la falta de relevancia e inversión en el sector rural y por ende en educación — problemáticas identificadas de antańo en la obra de Fals Borda-; la diferenciación que tienen los territorios en cuanto a cultura y formas de vida, los cuales no han sido suficientemente explorados; y la existencia de apuestas de investigación individuales preocupadas por aportar alternativas, que sin embargo, en su desarrollo resultan insuficientes.

Palabras clave: Rural, ruralidad, educación rural, territorio, entorno.

Recibido: $19 / 05 / 2021$ | Revisado: 25/05/2021

Aprobado: 08/09/2021 | Publicado: 31/12/2021 


\title{
How to understand the rural in rural education? A docu- mentary approach
}

\begin{abstract}
This article explores the conceptions of rural and rurality in rural education. The text focuses on the publications of the 2010 to 2020 years of the journal Praxis y Saber. At the same time, some theoretical references were used in order to broaden the instruments of understanding and inquiry. Thus, rurality was explored in a sociological context that compares development and education concerns; while rurality was approached as a broader and differentiated environment according to the experiences in different territories.

The survey shows as categories issues such as the lack of relevance and investment in the rural sector and therefore in education -problems identified in the work of Fals Borda-; the differentiation that territories have in terms of culture and ways of life, which have not been sufficiently explored; and the existence of individual research projects concerned with providing alternatives, which, however, in their development are insufficient.
\end{abstract}

Keywords: rural, rurality, rural education, territory, environment.

\section{En busca de lo rural en la educación rural}

Para entender cómo se comporta, transforma y se configura la escuela y la educación rural, es necesario preguntarnos por los conceptos que la componen, a saber, 'educación' y 'rural'. Empezaremos con el segundo de estos términos. En el contexto educativo ¿qué es lo rural?, ¿qué formas asume lo rural?, ¿qué transformaciones asume lo rural en combinación con la educación? y ¿qué efectos despliega lo rural en la educación? La categoría de lo rural emerge así, como plataforma de varias inquietudes pedagógicas y sociológicas que tratan de explicar en qué consiste ese sitio, espacio o escenario, qué características asume y qué aportes podría derivar el abordaje de estos interrogantes en la educación y los sujetos allí envueltos.

Orlando Fals Borda, en el libro El hombre y la tierra en Boyacá, nos ofrecerá pistas y herramientas para enfrentar estas inquietudes iniciales. A través del meticuloso trabajo de campo, Fals Borda adelanta una documentada observación de la sociedad rural de Boyacá y convierte su trabajo en una fuente ilimitada de reflexión para entender el presente complejo del departamento. Desde la revisión de los planteamientos y perspectivas de este sociólogo colombiano, pretendemos contribuir al debate en torno a las comprensiones de lo rural, con el propósito de aportar elementos para el abordaje de cuestiones como la migración, la pobreza de los sectores rurales, el menosprecio por lo campesino y su cultura, entre otros fenómenos recurrentes en la historia de los territorios. Fenómenos que nos invitan a preguntarnos por lo rural 
desde la misma ruralidad y no a la luz de modelos ajenos, como resulta cuando se estudia desde las preocupaciones derivadas del ámbito urbano.

Además de apoyarnos en las herramientas que nos ofrece Fals Borda, centraremos nuestra mirada en las concepciones y enunciados asociados a lo rural en las publicaciones de la revista Praxis \& Saber, corpus elegido para la presente revisión documental. Igualmente, será útil la lectura cruzada con otros autores relevantes en los temas de ruralidad y educación rural. Las publicaciones de la revista en el periodo 2010 a 2020, no solo nos han de aportar elementos teórico-conceptuales, sino que nos permitirá mapear las maneras como se apropia lo rural, los problemas que concentran la atención de los investigadores en el campo y las condiciones que le asocian quienes han dedicado parte de sus estudios a la educación rural o en la ruralidad.

\section{Una mirada a lo rural y la vida campesina desde Orlando Fals Borda}

Según Fals Borda (1957), si se desea entender lo rural es necesario revisar la población que allí habita, sus costumbres, condiciones de vida y relaciones con el entorno. Para ello, sitúa las posibilidades que ofrece el modelo científico con el fin de propiciar el diseño de instrumentos de planificación social. Esta cercanía con el método científico se debe no solo al momento histórico en el que se sitúan sus indagaciones, sino las intenciones mismas del autor en coherencia con la emergente sociología en Colombia. Pero, además, porque una de sus preocupaciones, en este caso en particular, se asocia con la educación y su planificación en los escenarios rurales en medio de las corrientes modernizadoras de la nación y las crecientes campañas de alfabetización. Es de recordar que para el periodo de trabajo de Fals Borda y de la expansión de los modelos desarrollistas en la región, las pésimas condiciones de los sistemas educativos locales y las abrumadoras tasas de analfabetismo se entienden como unas de las principales causas del 'atraso', pobreza y estancamiento de las economías nacionales. ${ }^{1}$

La mayor extensión del territorio colombiano se encuentra en el ámbito rural. Los espacios urbanos son reducidos en comparación con los rurales. Para la época de los estudios de Fals Borda, la mayor parte de la población colombiana se encontraba desperdigada a lo largo y ancho del país (aunque las ciudades, en sus pequeńos espacios, fuesen las zonas con mayor densidad poblacional). Es importante tener presente esta consideración, pues la atribución de la categoría de rural o urbano respondía a la concentración de la población en determinado territorio, lo cual, además de solapar la proporción poblacional de mayoría rural, ocultaba sus características por

1 Para ampliar las ideas respecto al funcionamiento de discursos como el desarrollismo y la modernización en la reconfiguración y dirección de las naciones, y su relación con el ordenamiento global gestado a partir de la segunda mitad del siglo XX, puede revisarse el trabajo de Arturo Escobar titulado La invención del tercer Mundo. Algunos otros elementos para la discusión son tratados en Espinel (2015a; 2015b). 
encontrarse supeditada a índices y políticas urbanas -tales políticas resultaban una contrariedad en un país eminentemente rural tanto en su territorio, como en su cultura y economía.

De hecho, uno de los criterios para definir a la población como rural, quizá el más determinante dentro de la planeación departamental, tenía que ver con la concentración de personas. Para la época, un municipio con más de 1.500 habitantes dejaba de ser rural para pasar a ser urbano. Boyacá, hacia los años sesenta, era el departamento más rural de Colombia con el 90,3 \% de su población en esta categoría, cifras que ya mostraba Fals Borda en 1957. Por otro lado, es importante anotar que actualmente se observa otra dinámica con referencia a la permanencia y disposición de la población rural departamental. Según la Gobernación de Boyacá (2018),

con una proyección de 1.287.032 millones de habitantes según cifras del DANE para el año 2020, distribuidos en 123 municipios, las tendencias por grupos de edad y sexo muestran notorias disminuciones de la población en las áreas rurales que tienen parte de explicación en la migración de la población joven a las cabeceras municipales y a las ciudades del departamento; aspecto relevante por su relación directa con oportunidades laborales y expectativas de mejora en la calidad de vida y educación (p. 8).

Este documento institucional también relaciona un crecimiento poblacional relativamente lento, con migraciones internas hacia los centros urbanos de Boyacá y hacia la capital del país por su cercanía geográfica.

El territorio rural se ha tomado en cuenta permanentemente desde las posibilidades que ofrece para el desarrollo económico y beneficio de la región. Se perfila además como un eje esencial para revisar la problemática medioambiental generada por la explotación de los recursos naturales por parte de las comunidades. Esta es una inquietud importante dentro de la gran mayoría de los proyectos rurales e investigaciones que ligan lo rural a la educación en la mayoría de casos en escenarios dentro del departamento de Boyacá. Según Pitta y Acevedo (2019), la importancia que adquiere la educación rural en relación con una perspectiva ambiental se debe primordialmente a la necesidad de resolver problemas en los territorios, entendidos desde su particularidad ambiental y desconocidos por quienes no actúan permanentemente en esos espacios. Por su parte, Figueroa y García (2019) plantean que es necesario incluir en los programas comunales la racionalidad ambiental, traducida en acciones para preservar los recursos naturales y culturales, y la preocupación por las relaciones con el medio ambiente, a fin de alinear las prácticas y procesos sociales en beneficio de la comunidad y el entorno.

Según Fals Borda (1957), en la mitad del siglo XX ya se observaba que una de las dificultades más recurrentes en el sector rural era el desperdicio de recursos naturales y la poca optimización de la tierra. Una de las propuestas que esbozaba el sociólogo 
para superar esta problemática era:

Fomento de un uso más económico de la tierra (especialmente de las vertientes) que proscriba el despilfarro de los recursos naturales y el desequilibrio en la subsistencia de los agricultores residentes, y del pastoreo, la irrigación, el drenaje y la forestación donde sea más conveniente (p. 217).

A medida que ha pasado el tiempo, los agricultores y mineros del sector rural boyacense han adoptado procesos más amigables con el medio ambiente, por un lado, porque las normas han sido más drásticas y por otro, porque han calado algunos programas educativos externos, impulsados por entes territoriales a través de las escuelas y colegios. Para Figueroa y García (2019), aunque la comunidad tiene un saber ambiental inoculado que invita a cuidar y conservar el territorio, se deben robustecer las iniciativas de formación y reflexión para mejorar el bienestar comunitario y lograr una producción más limpia y sostenible.

El alimento también es una preocupación latente dentro de la comunidad rural, no solo como producción y sustento económico, sino para el consumo propio. Algunos rastreos de comunidades indígenas, por ejemplo, muestran la preocupación de los habitantes por mantener el conocimiento sobre el resguardo de las semillas, los momentos propicios para la cosecha, la siembra, y la protección y reconocimiento de los alimentos autóctonos (Caicedo \& Espinel, 2018).

Figueroa y García (2019) y Pitta y Acevedo (2019) deducen que la integración de diversos aspectos de producción agrícola y el cuidado ambiental proporcionan sugerencias poderosas para el desarrollo de actividades agroecológicas, donde la escuela sirve de plataforma inicial para reforzar la política de la soberanía alimentaria. Los procesos de producción, transformación y consumo, dan paso a un proyecto sostenible y un consumo responsable que incluye el uso de la educación como dispositivo para mantener y activar estos saberes en niños y adolescentes.

La soberanía alimentaria se materializa, de este modo, en la capacidad de los agricultores para determinar y planificar una sostenibilidad anclada a iniciativas de desarrollo, lo que redunda en un beneficio importante para brindar independencia económica y dignidad a las poblaciones rurales. De ello se puede inferir que, la existencia de una conciencia comunitaria rural está directamente relacionada con el sentido de pertenencia al entorno, la preocupación y responsabilidad por el futuro del territorio, y la garantía de una subsistencia básica reflejada en el alimento.

Para Pitta y Acevedo (2019), lo rural está abocado a cumplir con la cobertura de la demanda alimentaria regional y nacional. La soberanía alimentaria es un dispositivo de compromiso y de derecho, que además pertenece a la política rural, la cual es analizada por la comunidad en múltiples espacios de discusión grupal. En consecuencia, se mantiene una relación estable entre la vereda y el entorno, a la vez que se conciben soluciones con mayor independencia de las disposiciones y control 
político urbano.

Conviene entonces enfatizar el sentido comunitario y de pertenencia con el territorio que ya desde mediados del siglo pasado identificaba Fals Borda. Aunque no muy desarrollado, el sentido de pertenencia e identidad territorial hace parte de la cultura campesina y ha trascendido a través del tiempo en el sector rural boyacense, lo cual puede aprovecharse para apalancar respuestas positivas a problemáticas actuales a través de, por ejemplo, proyectos originados desde las escuelas y colegios rurales. Sobre este arraigo Fals Borda (1997) menciona que:

empero el boyacense no es un hombre sin unidad, pues tiene áreas definidas de asociación e intercambio. En realidad, sus grupos ecológicos naturales recuerdan los de los Llanos Unidos, donde existe una dualidad formada por vecindarios rurales (open country neighborhoods) y centros de servicios o pueblos agrícolas. Puede avanzarse la opinión de que el vecindario boyacense, llamado comúnmente "vereda", es un conglomerado de familias que viven en fincas adyacentes y cuyos miembros no solamente tienen frecuentes contactos personales sino que han desarrollado una conciencia de identidad de grupo (p. 156).

De manera reciente, se han formulado progresivamente apuestas para atender las diferentes perspectivas de estudiantes de ambientes rurales, ante la convicción de que los entornos influyen directamente en las maneras de abordar las problemáticas. En esa línea, García y Furman (2014) indagan sobre las maneras que tienen los estudiantes para cuestionar sus formas de vivir el campo. El dejar la 'vergüenza' para preguntar, por ejemplo, es uno de los temas que emerge en investigaciones de este estilo. Estos temores de comunicación suelen ser recurrentes - aunque no exclusivos- en los estudiantes y poblaciones rurales. Generalmente se traducen en un posible miedo a la retaliación o ridiculización a partir de sus opiniones, lo cual resulta coherente con su etapa de desarrollo pero que, en opinión de algunas de las publicaciones revisadas, se acentúa en estos grupos poblacionales. Esto conlleva a que, al no ser compartidas las ideas, muchas veces se decida actuar de manera aislada e individual.

Al respecto, Fals Borda (1957) explica estas actitudes desde la relación con la forma en que se asentaron históricamente estas comunidades rurales.

Actualmente, desde el punto de vista histórico, podría decirse que el tipo de granjas dispersas, heredado de los mayores y confirmado en años de lucha contra la forma de colonización del blanco europeo, es una expresión del individualismo de los habitantes. El sistema de tenencia de la tierra, el tamańo de la propiedad, la fragmentación de la misma y el sistema agrícola han coadyudado a que se dispersen. Pero si se observa luego el funcionamiento de este tipo de poblamiento, habría que admitirse que promueve la falta de sociabilidad, el retraimiento, la reserva y la timidez. Es una de las causas de que 
el campesino piense más en sí mismo que en la comunidad, es decir, lo torna en un egocéntrico, lo confirma en su individualismo básico (pp. 205-206).

Además, desde la perspectiva de Fals Borda, el tamaño de la propiedad y del territorio al que se tenía acceso para la producción y la explotación, también era causa de la pobreza que vivía el departamento boyacense para la época.

Siguiendo adelante con el análisis, el tamaño de la propiedad es una causa de la concepción limitada del mundo que tienen los campesinos boyacenses, puesto que, siendo el minifundio una forma predominante, y ocurriendo en condiciones agrícolas, topográficas y edafológicas adversas, ha contribuido al desarrollo de la pobreza. Esta pobreza tiene consecuencias en la conducta: se refleja en la falta de educación, en elementos recreativos rutinarios, en deficiente alimentación, en descuido personal y de vestuario, en un bajo nivel de vivienda y en degradación moral y política (p. 205).

La pobreza en Boyacá ha sido predominante en el sector rural, excepto en casos puntuales de monocultivos y épocas de bonanza. De ello se ha derivado que parte importante de la población no tenga acceso o deserte frente a oportunidades de educación o promoción de sus potencialidades, lo cual, sumado a otros factores económicos, de cuidado y bienestar, conlleva al deterioro de las condiciones de vida digna. Según la Gobernación de Boyacá (2020), la situación de pobreza comparativamente es menor que en ańos anteriores y supera el índice de desigualdad nacional, sin embargo el nivel de pobreza en el sector rural en general es bastante alto. En busca de mejores oportunidades el campesino boyacense participa activamente en política, la cual representativamente no aportado nada significativo al campo, quizá como producto de ello, históricamente ha tenido la sensación cíclica de haber sido utilizado y subvalorado en el ámbito político nacional.

Enseguida, Fals Borda (1957) indica que esta situación es superable si se fortalecen algunas de las costumbres asociativas que se expresan alrededor del trabajo y la consecución de metas grupales, en pro del desarrollo veredal, las cuales redundan en el bienestar familiar.

Sin embargo, no es esta razón para pensar que el campesino boyacense no pueda participar en actos comunales. Ya se explicó que existen costumbres tales como el convite que dicen de una tendencia a la cooperación. El sentido cooperativo apenas está atrofiado; siendo resultado de un proceso educativo, puede perfectamente fortalecerse por medio de campañas y líderes hábiles (p. 206).

El papel de los líderes a los que se refiere Fals Borda ha sido suplido por años, en muchos casos, por los profesores, quienes se han comprometido con las poblaciones rurales. No obstante, son iniciativas, en su mayoría individuales, y generalmente dispersas; algunas de ellas están referenciadas en trabajos de investigación que bien 
podrían gestionarse, evaluarse y ser propuestas como insumos para los Proyectos Educativos Institucionales [PEI].

El análisis de las perspectivas de docentes y directivos sobre las necesidades y deficiencias de la escuela rural y el rol de los líderes en este contexto, hacen un aporte a los estudios de liderazgo escolar en Latinoamérica y, sobre todo, en el contexto particular de la educación rural colombiana (Acosta \& Deaza, 2019, p.17).

Los docentes acceden a información de primera mano sobre las problemáticas locales, mediante el contacto permanente con estudiantes y padres de familia; igualmente, conocen las necesidades estructurales que padece el sector rural y que terminan por afectar el entorno escolar. La relación con el contexto incide inevitablemente en la configuración del espacio y las dinámicas escolares. Lo mismo resalta Fals Borda en el marco de su investigación:

Las relaciones entre el hombre y la tierra en este departamento, como en muchos otros, dan pauta al ethos que permea a todo el grupo: se encuentran ellas en el corazón mismo de existencia diaria, dan significado a hechos del pasado y ofrecen una base para el mañana. Es aquí donde radica la importancia del examen de estas relaciones: como se expresa en la introducción, su estudio casi llega a constituirse en el más adecuado análisis de las propias bases sobre las cuales se funda no sólo la sociedad boyacense, sino la colombiana y la latinoamericana. (Fals Borda, 1957, p. 201).

El territorio, el entorno, el medio ambiente, terminan siendo influyentes -si no determinantes- en los comportamientos y relaciones de las comunidades rurales. Las expectativas de progreso y las políticas estatales han determinado la evolución en la forma de afrontar las problemáticas, que infortunadamente son, muchas veces, iniciativas impulsadas por esfuerzos individuales, huérfanos de una política estatal seria y pertinente.

Todo ello hace que, fácilmente, los territorios rurales se conviertan en escenarios de violencia, pobreza y abandono. Empero, las problemáticas propias e inquietudes locales sobre su propio territorio son desconocidas y opacadas por políticas públicas delineadas desde -y para- los grandes centros urbanos. Vale la pena resaltar que $E l$ hombre y la tierra en Boyacá, que hemos retomado como interlocutor, es un trabajo realizado por Fals Borda hace casi siete décadas y, sin embargo, se observa la misma correlación entre la falta de interés del Estado y la cultura de supervivencia del campesino boyacense con fuertes arraigos sociales y sentimentales por su entorno.

\section{Ruralidad. Nuevos Horizontes de lo Rural}

La preocupación de los últimos ańos alrededor del desenvolvimiento de la educación rural, ha girado en torno a las particularidades que encontramos en 
territorios con diferentes formas de pensar, vivir y sobrevivir en el campo. Ese ser rural está permeado por la cultura circundante, la política, la religión entre otra serie de elementos no visibles, pero presentes en la relación de los sujetos con sus territorios. Podríamos decir que la ruralidad conjuga lo físico del territorio con otros aspectos sociológicos que influyen dentro de este.

La ruralidad es ese hábitat construido durante generaciones por la actividad agropecuaria, es el territorio donde este sector ha tejido una sociedad. Este concepto incorpora una visión multidisciplinaria que reivindica los aspectos antropológicos, sociopolíticos, ecológicos, históricos y etnográficos, además de la tradicional visión económica de lo agropecuario y de lo rural y es abiertamente alternativo a la visión sectorial que predomina en las estrategias de política rural de nuestros países (Echeverri \& Ribero, 2002, p. 26).

Según Buitrago-Bonilla (2012), hoy en día, la ruralidad se plantea como un patrimonio territorial que da sentido a un estilo de vida particular, pero que es diferente a la concepción tradicional, debido a la relación que se establece entre la sociedad global y las comunidades. Por tal motivo, los fenómenos sociales, producto de los vínculos entre lo urbano y rural, lo local y lo global, son fundamentales dentro de esta nueva idea. Herrera y Buitrago (2015) consideran necesario avanzar en trabajos que den cuenta de las condiciones, riquezas, necesidades y proyectos dentro de contextos rurales, para favorecer la generación de conocimiento y abrir una discusión pertinente en la formación de los docentes, la política educativa y el marco regulatorio local y nacional respecto al territorio.

La profesora Diana Soto realiza un profundo recorrido histórico de la ruralidad desde la óptica de la mujer maestra en la época de la violencia bipartidista en Colombia. En un primer análisis, Soto (2013) indaga por la relación de las zonas rurales y urbanas a través de una visión transversal de la violencia de partidos de los años 1950 en el país. La realidad de la guerra ha cambiado los pensamientos, la sociedad, la cultura y el enfoque educativo, no obstante, observa Soto, la problemática en marcos de violencia sigue siendo muy parecida época tras época. Cabe destacar, no solo los profundos cambios que trajeron consigo la violencia y el desplazamiento forzado, sino el lugar que fue asumiendo paulatinamente la educación como principal elemento de superación socio-económica.

De otra parte, Buitrago-Bonilla (2018) destaca que dentro de diferentes percepciones de ruralidad existen diversas dinámicas de la vida rural, donde interviene intermitentemente la comparación con lo urbano.

Otro aspecto fundamental se refiere al potencial que tienen las ruralidades, el cual debe ser reconocido y abordado desde procesos de investigación, tratando de hacerlo sin sesgo urbano. Además de ello, es vital que los currículos de los programas de formación docente asuman el estudio de las ruralidades como 
imprescindible. Desde esta mirada, si bien es cierto que en Colombia se han implementado programas y políticas relacionadas con diversos aspectos de las ruralidades, aún no son suficientes los esfuerzos y el sector, en especial desde la educación, requiere mayor atención (p. 15).

El trabajo del profesor Rafael Buitrago genera la discusión de la ruralidad y las ruralidades entendidas como diferentes formas de ver y sentir lo rural, como un patrimonio de orden territorial alejado de las antiguas concepciones y en constante relación con la sociedad global. Su trabajo se concentra en el contexto boyacense, lo cual brinda cercanía conceptual con el medio investigado aquí. Estudios como los explorados en este acercamiento documental muestran la necesidad de seguir profundizando desde el sector educativo para develar qué aportes o qué papel cumple la educación dentro de las ruralidades y, a su vez, qué posibilidades aporta lo rural en la educación.

Por su parte, Lara y Pulido (2020) destacan las características especiales que ofrece la ruralidad y que favorecen el desarrollo de investigaciones sobre cómo se desenvuelve el estudiante, en contextos de la escuela rural, y cuáles son sus comportamientos frente a cuestionamientos profundos. El escenario configurado por la escuela, el maestro y la comunidad nutre proyectos como Filosofía e infancia: investigación y posibilidades de innovación en la escuela rural, objeto de estudio de los autores del artículo; lo cual posibilita diferentes ópticas y enriquece las perspectivas de análisis frente a la educación rural y la escuela.

Los artículos revisados coinciden en enfatizar sobre la necesidad de profundizar en la formación pertinente de los maestros rurales. Así mismo, se plantea de manera urgente asociar la gestión y la política pública con el rol de los docentes rurales: "en conclusión, es abordar una política pública que tenga en cuenta las ruralidades del país, los planteamientos de la nueva ruralidad, la investigación y el cambio en la formación de los formadores" (Arias et al., 2008, p. 2). Arias et al. también plantean un programa integral que tenga en cuenta los diferentes actores que intervienen en la escuela rural, con el fin de generar una mejor articulación y reconocimiento de las ruralidades en relación con la educación.

Según Camarda (citado por Molina \& Mesa, 2019) en las ruralidades se presta poca atención a la heterogeneidad de las comunidades, las necesidades ambientales y el desarrollo del trabajo productivo. Atendiendo estas inquietudes, algunas iniciativas se han preocupado por darle cabida a la actividad científica y cultural en el sector rural. Varios de estos programas a nivel nacional han decantado en proyectos pertinentes desde el sector educativo. Plata (2016), revisa el impacto del programa ONDAS de Colciencias en el departamento de Boyacá, que es una estrategia que tiene como objetivo promover el interés de niños y jóvenes por la investigación y desarrollar en ellos actitudes y habilidades investigativas, lo cual los integra activamente en la ciencia, la cultura, la tecnología e innovación, aunque su ámbito de aplicación no 
exclusivamente rural, ha arropado este sector con excelentes resultados.

De otra parte, queda para revisar algunas apuestas que se han tratado de introducir en la educación, sobre todo en primaria y media rural, a causa de las medidas de confinamiento masivo como efecto de la crisis sanitaria global. Una de esas estrategias la educación virtual o remota, develó la deficiente red tecnológica en las zonas más apartadas de Colombia, por ello, al no contar con las herramientas necesarias para seguir este modelo educativo promovido durante la coyuntura sanitaria, esta iniciativa no pudo cumplir con las expectativas educativas. Los docentes, conocedores de las condiciones y características locales, durante el aislamiento terminaron usando alternativas ya probadas en otros tiempos como el correo escrito y la comunicación radial.

\section{Educación rural. Una nueva apuesta}

Para entender cómo se analiza la educación rural bien podríamos empezar por observar qué sucede en el sitio propio donde se desarrolla ese primer contacto de educación, es decir, la escuela. A propósito Pulido (2018) señala:

la escuela es el espacio, a través de sus diversos artefactos, donde las producciones del mundo son puestas en público, además de ser susceptibles de ser reproducidas, cuestionadas o validadas. Por eso se convierten en objetos de estudio e investigación. Lo escolar se convierte en el lugar y el tiempo del estudio y de la práctica. Aunque está fuera de la sociedad, estudia lo que pasa en la sociedad. La escuela, a diferencia de lo que los críticos plantean, no se cierra sino que se abre al mundo, pues es la realidad exterior la que va a la escuela representada en sus actores y las situaciones que ellos mismos construyen (p. 10).

La escuela es un lugar o, más bien, un espacio especial donde el sujeto toma distancia del mundo para hacerlo objeto de estudio y reflexión. Un lugar público pero a la vez íntimo, de desarrollo intelectual y construcción de lo común. Una escuela rural es un lugar de confluencia de expectativas y, usualmente, un punto céntrico de encuentro que acoge y que, por ello, es valorado por la comunidad.

$\mathrm{Al}$ abordar el desarrollo de lo rural y la ruralidad, entendemos también los procesos que ha enfrentado la educación rural. Desde sus inicios, la escuela rural e indígena ha sido proscrita y supeditada a prácticas colonialistas que intentaron imponerse sobre los vestigios culturales de los territorios conquistados, inhóspitos y distantes de las ciudades (Caicedo \& Espinel, 2018). Lo anterior ha sucedido, entre otros territorios, en el departamento de Boyacá, que fue epicentro de la colonización española. Vale la pena señalar que, recientemente se ha retomado el estudio de los aportes indígenas a la educación rural de orden nacional con propuestas como el Plan Especial de Educación Rural [PEER], la tradición oral, el cuidado e integración con el medio ambiente, los conocimientos ancestrales, en el que también las comunidades raizales, 
negras y campesinas ocupan un importante espacio por ser residentes permanentes de los sectores rurales y quienes han sufrido la violencia histórica en el país, en defensa de estas tradiciones.

Antes de este plan estuvieron también las propuestas del Plan Educación Rural [PER] del año 2000, la Escuela Nueva o Escuela Activa desde la década de 1970. También encontramos propuestas como la etnoeducación, el Servicio Educación Rural [SER] y el Servicio de Aprendizaje Tutorial [SAT], estos últimos de la década de 1990. Estas propuestas de orden nacional y aplicación local demuestran que se han planteado diversas iniciativas de formación, las cuales no han profundizado o recogido en su totalidad la visión educativa del estudiante rural y, por lo tanto, no han mantenido su impacto, ni mostrado resultados de largo plazo. Es importante resaltar que apuestas y proyectos como los mencionados están sujetos a la voluntad política y la poca inversión económica.

Por su parte Ortiz y Betancourt (2020), en su trabajo acerca del Plan de Aceleración de Aprendizaje $[\mathrm{PAA}]^{2}$, concluyen aspectos determinantes para la aplicación de este plan en zonas apartadas, y de postconflicto, cuyo modelo se ocupó del desarrollo de habilidades y destrezas sobre los conocimientos a partir de la realidad concreta del sujeto. Este proyecto se destacó por brindar la oportunidad de escolarización a personas en extraedad. Las evaluaciones hechas al programa mostraron que buena parte de los estudiantes que ingresaron a la escuela mediante el PAA lograron terminar el nivel de primaria con un rendimiento similar al de los estudiantes que terminaban en un aula regular para este ciclo. Sin embargo, al iniciar secundaria hubo bastante deserción de los estudiantes adscritos al programa, esta se debió por un lado a la extraedad y por otro a las condiciones adversas por las que atraviesan las escuelas que atienden a poblaciones rurales, indígenas marginales, poca inversión en infraestructura escolar, falta de insumos educativos, excesiva rotación y ausentismo de docentes y falta de capacitación para los procesos de aprendizaje.

La educación rural puede asumirse desde un papel que reivindique a las comunidades, potencie el entramado social y resalte las especificidades de las regiones para superar la tutela urbanocéntrica.

En sintonía con lo anterior, Marco Raúl Mejía (2015) afirma que, "a partir de las particularidades de las sociedades sudamericanas, la educación popular pretende crear un proyecto desde el sur para proponer una manera distinta de comprender la situación actual latinoamericana alejada de las clásicas explicaciones eurocentristas” (p.98). En este sentido, se propone la necesidad de crear una educación que permita el desarrollo local, conserve las potencialidades del origen e invite a reflexionar sobre

2 El PAA (plan acelerado de aprendizaje) del MEN, es un modelo busca apoyar a niños, niñas y jóvenes de la básica primaria que están en extraedad, con el fin de que amplíen su potencial de aprendizaje, permanezcan en la escuela y se nivelen para continuar exitosamente sus estudios. Fortaleciendo la autoestima, la resiliencia, enfocándolos a construir su proyecto de vida 
la posibilidad de ser inventores y no repetidores.

Asimismo, para Lara y Pulido (2020) las escuelas rurales se convierten en el centro de la actividad comunitaria, porque pueden establecerse, determinarse, criticarse, resistirse o separarse de la vida y la supervivencia; también despiertan las emociones, sentimientos y expresiones de la escritura a partir de la experiencia propia del contexto y la posibilidad de ser de otras formas. Morales y Pulido (2018) y Torres et al. (2017) proponen la posibilidad de generar pensamiento crítico en los estudiantes rurales, a través de la lectura de diversas ópticas y percepciones que les permiten desarrollar creatividad y visibilizarse como creadores de historias propias, sujetos que indagan, analizan y plantean soluciones centradas en sus realidades.

De otra parte, Zamora y Mendoza (2019) insisten en que, además del estudiante, se debe identificar qué sucede con el profesor rural en su rol formativo, su relevancia y la manera como se desenvuelve. En este sentido, proponen dos asuntos idóneos para discutir desde la academia. Un primer elemento es que, en la educación rural tal como está pensada desde la política pública con perspectivas urbanas, no es posible cimentar una identidad didáctica que dé cuenta de los asuntos particulares de una escuela que es organizacionalmente distinta a las de las ciudades, y, de otro lado, tampoco es viable imponer la autonomía como efecto de presión y rigidez en la estructura escolar, pues lo rural desdeña otras formas de aprender y acceder al conocimiento. Los autores también señalan que los saberes no pasan por las inquietudes de los estudiantes, ya que son relacionados y enseñados desde un escenario ajeno y foráneo —incluso para el maestro-.

Por último, en el corpus documental revisado también se abre una discusión sobre la igualdad pretendida en derechos de aprendizaje idénticos para todo el país, pues estos pueden convertirse en un elemento homogeneizador que le resta autonomía al maestro sobre su hacer y elimina la posibilidad de discernir sobre la pertinencia de los contenidos y objetivos entendidos desde los 'resultados de aprendizaje'. Estándares que además de fijar, encasillan, masifican y anulan la diferencia.

\section{Conclusiones}

El análisis desde Fals Borda devela que las problemáticas del sector rural persisten más de medio siglo después de la publicación del libro estudiado en este artículo. Podría decirse que los intentos por desarrollar una mejor y digna vida al sector campesino no han alcanzado los resultados esperados, razón por la cual, la educación tiene que revisar su pertinencia e impacto en el medio.

El concepto de 'lo rural' está ligado a su contraparte, 'lo urbano', por lo que algunos análisis parten de esa dicotomía. Diversos autores proponen que 'lo rural' se analice como un concepto unitario y sea desligado de 'lo urbano', mientras que otros permanentemente hacen la comparación con el fin de mostrar las diferencias 
en la perspectiva o trato que se les da desde el punto de vista de reconocimiento, inversión y relevancia. Claramente, la identificación de las problemáticas rurales en Boyacá se ha llevado a cabo desde hace muchos años, no obstante, además de cíclicas, el departamento continúa sin políticas claras ni programas a largo plazo. Existen propuestas desde el ámbito investigativo, pero por el momento no se traducen en programas sólidos, y muchas de ellas permanecen aisladas, debilitadas.

De otra parte, se destaca la existencia de dos preocupaciones reiterativas en los artículos de la revista Praxis y Saber entre los años 2010 a 2020 que abordan lo rural. El primero de ellos trata la cuestión medioambiental y el segundo, el tema alimentario o de producción de subsistencia económica. Igualmente, la ruralidad y nueva ruralidad emergen como conceptos que explican la diversidad de costumbres, necesidades y posibilidades de los territorios, sin embargo, en ocasiones carece de profundad temática. En este sentido, se muestra un campo de acción amplio para fortalecer y reconocer la diversidad y la potencia del sector rural y las ruralidades.

Es importante revisar que sucede con 'lo rural', 'la ruralidad' y 'la nueva ruralidad' desde la óptica del investigador y el conjunto de las investigaciones. Además, por las condiciones, contextos y poblaciones que la habitan, la Universidad Pedagógica y Tecnológica de Colombia, que desarrolla su trabajo formativo principalmente en Boyacá, está llamada a ubicarse como centro de estudios alrededor de lo rural y las ruralidades. Revisar las investigaciones que allí se desarrollan puede dar pautas para proponer una educación que reconozca y potencie ambientes singulares, propios y originarios.

\section{Referencias}

Acosta, L. M., \& Deaza, L. S. (2019). Liderazgo Escolar: experiencias de directivos y profesores para la justicia social en la escuela rural [Tesis de maestría, Universidad de La Sabana]. https://intellectum.unisabana.edu.co/handle/10818/38384

Arias, J. M., Perdomo, L., Silva, G., \& Parra, F. (2008). Caminos para crear un nuevo mundo rural. Al tablero, (45). http://www.mineducacion.gov.co/1621/ article-168266.html

Buitrago-Bonilla, R. E. (2018). Transformaciones sociales educativas desde procesos investigativos [Editorial]. Praxis \& Saber, 9(21), 9-20. https://doi. org/10.19053/22160159.v9.n21.2018.8922

Caicedo, S. L., \& Espinel, O. (2018). Educación indígena Kamëntzá. Crianza, pensamiento, escucha. Praxis \& Saber, 9(20), 15-40. https://doi. org/10.19053/22160159.v9.n20.2018.8294

Espinel, O. (2015a). El régimen de los derechos humanos. Diseños globales, subordinación y colonialidad. En H. Vásquez \& L. Siri (comp.), Representaciones discursivas de la violencia, la otredad y el conflicto social en Latinoamérica (pp.13- 
28). Universidad de Buenos Aires.

Espinel, O (2015b). Educación para el ¿¿desarrollo? El sofisma de la modernización y el desarrollo. Espacios en Blanco. Revista de Educación, (25), 333-349. http:// digital.cic.gba.gob.ar/handle/11746/10296

Echeverri, R., \& Ribero, M. (2002). Nueva ruralidad. Visión del territorio en América Latina y el Caribe IICA; CIDER; Corporación Latinoamericana Misión Rural.

Fals Borda, O. (1957). El hombre y la tierra en Boyacá. Ediciones documentos colombianos.

Figueroa, D., \& García, L. (2019). Comprensión de las acciones comunitarias en educación ambiental en Chiquinquirá, Boyacá. Praxis \& Saber, 10(23), 293-314. https://doi.org/10.19053/22160159.v10.n23.2019.9735

Herrera, L., \& Buitrago, R.(2015). Educación rural en Boyacá, fortalezas y debilidades desde la perspectiva del profesorado. Praxis \& Saber, 6(12), 169-190. https://doi. org/10.19053/22160159.3768

García, S., \& Furman, M. G. (2014). Categorización de preguntas formuladas antes y después de la enseñanza por indagación. Praxis \& Saber, 5(10), 75-91. https:// doi.org/10.19053/22160159.3023

Gobernación de Boyacá. (2018). Ordenamiento territorial departamental de Boyacá. https:/www.dapboyaca.gov.co/wp-content/uploads/2018/09/ DIN\%C3\% 81 MICAS-DE-OCUPACI\%C3\%93N-DE-SUELO-CONRELACI\%C3\%93N-A-LOS-ASENTAMIENTOS-HUMANOS.pdf

Gobernación de Boyacá. (2020). Plan de desarrollo 2020-2023. https://www.boyaca. gov.co/wp-content/uploads/2020/04/pddBoyaca2v.pdf

Lara, P. A., \& Pulido, O. (2020). Escritura como práctica de sí y escuela rural. Praxis \& Saber, 11(25), 23-47. https://doi.org/10.19053/22160159.v11.n25.2020.10480

Mejía, M. R. (2015). La educación popular en el siglo XXI. Una resistencia intercultural desde el sur y desde abajo. Praxis \& Saber, 6(12), 97-128. https:// doi.org/10.19053/22160159.3765

Molina, L. E., \& Mesa, F. Y. (2019). Las TIC en escuelas rurales: realidades y proyección para la integración. Praxis \& Saber, 9(21), 75-98. https://doi. org/10.19053/22160159.v9.n21.2018.8924

Morales, L., \& Pulido, O. (2018). Ambientes filosóficos para la lectura en la escuela rural. Praxis \& Saber, 9(21), 99-124. https://doi.org/10.19053/22160159. v9.n21.2018.8925

Ortiz, L. M., \& Betancourt, C. (2020). Evaluación del Programa de Aceleración del Aprendizaje: una apreciación estratégica hacia la educación inclusiva en el 
posconflicto. Praxis\&Saber, 11(25), 97-110.https://doi.org/10.19053/22160159. v11.n25.2020.8207

Pitta, M. J., \& Acevedo, Á. (2019). Contribuciones de la agroecología escolar a la soberanía alimentaria: caso Fundación Viracocha. Praxis \& Saber, 10(22), 195221. https://doi.org/10.19053/22160159.v10.n22.2019.8839

Plata, M. E. (2016). Formación en investigación en el departamento de Boyacá: aportes del programa Ondas - Colciencias. Praxis \& Saber, 7(15), 103-125. https://doi.org/10.19053/22160159.v7.n15.2016.5725

Pulido, O. (2018). ¡Hay que defender la escuela![Editorial]. Praxis \& Saber, 9(20). 9-14. https://doi.org/10.19053/22160159.v9.n20.2018.8426

Soto, D. E. (2013). La ruralidad en la cotidianidad escolar colombiana. Historia de vida de la maestra rural boyacense 1948-1974. Revista Historia de la Educación Latinoamericana, 14(18), 211-243. https://doi.org/10.19053/01227238.1621

Torres, D. L., Fonseca, W. P., \& Pineda, B. N. (2017). Las vivencias como estrategia de fortalecimiento del pensamiento crítico en educación rural. Praxis \& Saber, 8(17), 201. https://doi.org/10.19053/22160159.v8.n17.2018.7207

Zamora, L. F., \& Mendoza, A. P. (2019). La formación de educadores para el trabajo rural: el reto planteado por la escuela rural multigrado en Colombia. Nodos y Nudos, 6(45), 74-87. 